\title{
Impact of nanosizing on dermal delivery and antioxidant activity of quercetin nanocrystals
}

\author{
Maria Letizia Manca ${ }^{\mathrm{a}}$, Francesco Lai ${ }^{\mathrm{a}, *}$, Rosa Pireddu ${ }^{\mathrm{a}}$, Donatella Valenti ${ }^{\mathrm{a}}$, Michele Schlich ${ }^{\mathrm{a}}$, \\ Elena Pini ${ }^{\mathrm{b}}$, Giorgia Ailuno ${ }^{\mathrm{c}}$, Anna Maria Fadda ${ }^{\mathrm{a}}$, Chiara Sinico ${ }^{\mathrm{a}}$ \\ ${ }^{a}$ Department of Life and Environmental Sciences, Unit of Drug Sciences, University of Cagliari, Cagliari, 09124, Italy \\ b Department of Pharmaceutical Scienc, Sezione di Chimica Generale e Organica "A.Marchesini', Università Degli Studi di Milano, Milano, Italy \\ ${ }^{\mathrm{c}}$ Dipartimento di Farmacia (DIFAR), University of Genova, Genova, 16148, Italy
}

\section{A R T I C L E IN F O}

\section{Keywords:}

Quercetin

Nanosuspension

Nanocrystal

Keratinocytes

Antioxidant activity

DSC

XRPD

FTIR

\begin{abstract}
A B S T R A C T
Quercetin is one of the most effective natural compounds against skin disorders related to inflammatory and oxidative processes. However, its efficacy is generally limited by its low bioavailability mainly due to the poor water solubility. In this work, quercetin was formulated as nanosuspension in order to investigate whether the nanosizing approach could improve both its saturation solubility and cutaneous bioavailability. Quercetin nanosuspensions at two different concentrations ( 3 and 5\%), were obtained by a wet media milling technique using Tween 80 and Poloxamer 188 as stabilizers. The obtained nanocrystals were deeply characterized by using different techniques such as Scanning Electron Microscopy, Differential Scanning Calorimetry, X-Ray Powder Diffractometry, Fourier Transform Infrared Spectroscopy and Photon Correlation Spectroscopy.

Quercetin nanocrystals exhibited a mean diameter ranging between 326 and $474 \mathrm{~nm}$ and a polydispersity index lower than 0.30 . Moreover, the size reduction greatly improves quercetin solubility and dissolution rate, thus promoting its accumulation in the different skin layers. Finally, in vitro studies using keratinocytes showed the high biocompatibility of nanosuspensions and their ability to counteract the oxidative effect of hydrogen peroxide on cells, suggesting their possible use for the treatment of skin disorders.
\end{abstract}

\section{Introduction}

Polyphenols, such as flavonoids, are potent antioxidants commonly used for topical applications as a defence against natural skin damage (photo-ageing), skin cancer prevention, and skin care [1-3].

In particular, quercetin $\left(3,3^{\prime}, 4^{\prime}, 5,7\right.$-pentahydroxyflavone), is considered one of the most powerful natural antioxidant and most common dietary polyphenol widely found in different plants including vegetables, fruits, medicinal herbs and red wine. This flavonoid has been intensively investigated mainly because of its well-known anti-inflammatory, antioxidant, antiviral, anticarcinogenic and cardioprotective properties [4].

Despite of these promising therapeutic properties, biomedical applications of quercetin are severely hampered by its low skin bioavailability due to its poor water solubility. To overcome this limit, many synthetic [5] or technological approaches have been developed including different drug delivery systems. Among all, conventional and modified liposomes and niosomes, have been used aiming at improving the skin delivery of quercetin and other natural and synthetic substances [6-9].

However, in the last years, a great attention has been devoted to drug nanocrystal technologies, which are considered one of the most successful approaches to formulate poorly soluble drugs [10,11].

Nanocrystals can be defined as nanoparticles made of pure drug, with no matrix material, and an average diameter below $1 \mu \mathrm{m}$ (typically in the range of $200-500 \mathrm{~nm}$ ). They can be prepared in both water and non-water liquid phase as colloidal suspension (nanosuspension).

Nanosuspensions can be stabilized using ionic stabilizers (sodium dodecyl sulphate, lecithin, etc.) or non-ionic surfactants or polymers (poloxamers, polyethylene glycol, polysorbates, polyvinylpyrrolidone, cellulose polymers, etc.).

Basically, nanosuspensions can be prepared by bottom up or top down technologies or by a combination of both [12]. The top down technology is based on particle disintegration techniques including high pressure homogenization [11,13] and wet media milling technique $[14,15]$. In the bottom up technology, the low water-soluble drugs are dissolved in a solvent and then precipitated in different ways as nanocrystals in a surfactant solution [16].

\footnotetext{
${ }^{*}$ Corresponding author. Department of Life and Environmental Sciences, Unit of Drug Sciences, University of Cagliari, Via Ospedale 72, 09124, Cagliari, Italy.

E-mail address: frlai@unica.it (F. Lai).
} 
In vitro and in vivo studies have demonstrated that nanocrystals can increase dermal drug bioavailability by enhancing the dissolution rate and saturation solubility, thus, leading to an increased concentration gradient between dermal formulation and the skin and, consequently, an improved skin penetration of drugs [12,17-19]. Moreover, the enhanced retention time of these systems on the skin, also through accumulation in the hair follicles [20], favours a prolonged and intimate interaction between drug and skin and promoting its therapeutic effect.

Given that, in the present study, quercetin nanosuspensions were prepared by means of the wet media milling technique. The solid state of quercetin nanocrystals was investigated by using different technologies such as scanning electron microscopy (SEM), differential scanning calorimetry (DSC), X-ray powder diffractometry (XRPD), Fourier transform infrared (FTIR) spectroscopy and Photon Correlation Spectroscopy (PCS) and compared with that of the coarse/bulk drug.

The ability of nanosuspensions to promote the skin delivery of quercetin was evaluated in vitro by using Franz diffusion cells and pig skin. Finally, the antioxidant activity of quercetin nanocrystals was tested by assessing its ability to scavenge free 2,2-diphenyl-1-picrylhydrazyl (DPPH) and to protect human keratinocytes in vitro against hydrogen peroxide damages.

\section{Material and methods}

\subsection{Materials}

Tween 80 (T80) was purchased from Galeno (Comeana, Italy). Lutrol $^{\circledR}$ F68 (Poloxamer 188, P188) was a gift from BASF AG (Ludwigshafen, Germany). Quercetin and all the other reagents were of analytical grade and were purchased from Sigma-Aldrich (Milan, Italy). Cell medium, foetal bovine serum, penicillin, and streptomycin and all the other materials for cell culture were purchased from Life Technologies Europe (Monza, Italy).

\subsection{Preparation of coarse suspensions}

Coarse drug suspensions were prepared by dispersing quercetin at two different concentrations ( 30 or $50 \mathrm{mg} / \mathrm{ml}$ ), in a water solution containing P188 or T80 (Table 1). Dispersions were homogenised by using an Ultra Turrax T25 basic (IKA, Werke) for $5 \mathrm{~min}$ at $6500 \mathrm{rpm}$.

\subsection{Preparation of nanosuspensions}

Nanosuspensions were prepared using the wet media milling technique. Briefly, quercetin ( 30 or $50 \mathrm{mg} / \mathrm{ml}$ ) was dispersed in an aqueous solution containing P188 or T80 (10 mg/ml) using an Ultra Turrax T25 basic for $5 \mathrm{~min}$ at $6500 \mathrm{rpm}$. The obtained coarse suspension was divided into conical microtubes containing $\sim 0.4 \mathrm{~g}$ of $0.1-0.2 \mathrm{~mm}$ yttrium-stabilized zirconia-silica beads (Silibeads ${ }^{\oplus}$ Typ ZY Sigmund Lindner, Germany). The microtubes were maintained under constant oscillation at $3000 \mathrm{rpm}$ using a beads-milling cell disruptor equipment (Disruptor Genie, Scientific Industries, USA). The obtained nanosuspensions were separated from the milling beads by sieving and characterised.

\subsection{Solubility studies}

Quercetin water solubility was measured for both bulk quercetin and nanosuspensions. Formulations were kept under constant stirring for $72 \mathrm{~h}$ in a thermostatic bath at $37{ }^{\circ} \mathrm{C}$. At preselected time intervals, samples were withdrawn and centrifuged. Clear supernatants were diluted with methanol and analysed by HPLC for quercetin content. Solubility studies were performed in triplicate.

\subsection{HPLC analysis}

Quercetin content was determined by high performance liquid chromatography (HPLC) after dilution of the samples with methanol. The analysis of solutions were performed at 255 and $367 \mathrm{~nm}$ using a chromatograph Alliance 2690 (Waters, Milan, Italy) equipped with a fluorimeter detector and a computer integrating apparatus (Empower 3). The column was a SunFire C18 $(3.5 \mu \mathrm{m}, 4.6 \times 150 \mathrm{~mm})$, and the mobile phase was a mixture of acetonitrile, water and acetic acid (94.8:5:0.2, v/v), delivered at a flow rate of $1.0 \mathrm{ml} / \mathrm{min}$. A standard calibration curve was built up by using working standard solutions. Calibration graphs were plotted according to the linear regression analysis, which gave a correlation coefficient value (R2) of 0.999 .

\subsection{Nanocrystal characterization}

The average diameter and polydispersity index (PI) of both quercetin coarse suspensions and nanosuspensions, were determined by Photon Correlation Spectroscopy (PCS), using a Zetasizer nano-ZS (Malvern Instruments, Worcestershire, United Kingdom). Samples were backscattered by a helium-neon laser $(633 \mathrm{~nm})$ at an angle of $173^{\circ}$ and a constant temperature of $25^{\circ} \mathrm{C}$. Zeta potential was estimated using the Zetasizer nano-ZS by means of the M3-PALS (Mixed Mode Measurement-Phase Analysis Light Scattering) technique, which measures the particle electrophoretic mobility. Nanosuspensions stability study was assessed by monitoring the average size, the homogeneity of the systems and their surface charge over 90 days of storage at room temperature $\left(25 \pm 1{ }^{\circ} \mathrm{C}\right)$.

\subsection{Solid state characterization}

XRPD analyses were carried out by using a Rigaku Miniflex diffractometer. The setting parameters were Ni-filtered $\mathrm{CuK \alpha}$ radiation detector $(\lambda=1.5405 \AA)$, voltage of $30 \mathrm{kV}$, current of $15 \mathrm{~mA}$, scan angular speed $2^{\circ} / \mathrm{min}$ and scan step time $2.00 \mathrm{~s}$ in the $2 \theta$ range from $3^{\circ}$ to $60^{\circ}$. The XRPD patterns of quercetin raw material, freeze dried quercetin nanocrystal and P188 were recorded.

FT-IR spectra were collected using a Spectrum One PerkinElmer (MA, USA) FT-IR Spectrometer in the spectral region between 4000 and 600 or $450 \mathrm{~cm}^{-1}$ for solid or liquid compounds, respectively, and analysed by transmittance technique with 32 scans and 4 or $8 \mathrm{~cm}^{-1}$ resolution. Solid samples were mixed in a mortar with $\mathrm{KBr}(1: 100)$ and pressed by a hydraulic press (10 tons) into small tablets. For liquid samples one drop was placed between two plates of sodium chloride.

The DSC curves of solid samples were recorded on a PerkinElmer DSC 6 differential scanning calorimeter calibrated with indium. The thermal behavior was studied by heating $2.5 \mathrm{mg}$ samples in aluminum

Table 1

Composition of quercetin nanosuspension (NS) and coarse suspension (CS) stabilized using Poloxamer 188 (P188) or Tween 80 (T80).

\begin{tabular}{|c|c|c|c|c|}
\hline & Quercetin (mg) & Poloxamer188 (mg) & Tween80 (mg) & Water (ml) \\
\hline NS3 P188/CS3 P188 & 30 & 10 & & 960 \\
\hline NS5 P188/CS5 P188 & 50 & 10 & & 940 \\
\hline NS3 T80/CS3 T80 & 30 & - & 10 & 960 \\
\hline NS5 T80/CS5 T80 & 50 & - & 10 & 940 \\
\hline
\end{tabular}


crimped pans under nitrogen gas flow.

\subsection{In vitro skin delivery studies}

Experiments were performed under non-occlusive conditions using vertical Franz diffusion cells (diffusion area $0.785 \mathrm{~cm}^{2}$ ) and newborn pig skin. One-day-old Goland-Pietrain hybrid pigs (about $1.2 \mathrm{~kg}$ ) were provided by a local slaughterhouse. The skin ( $\mathrm{n}=6$ per formulation), stored at $-80{ }^{\circ} \mathrm{C}$, was pre-equilibrated in saline solution at $25{ }^{\circ} \mathrm{C}$ for $12 \mathrm{~h}$ and then sandwiched between donor and receptor cells. The receptor was filled with $5.5 \mathrm{ml}$ of saline solution, thermostated at $37 \pm 1{ }^{\circ} \mathrm{C}$ and continuously stirred. Samples $(100 \mu \mathrm{l})$ were applied onto the skin surface, and at regular intervals, up to $24 \mathrm{~h}$, the receiving solution was withdrawn, replaced with pre-thermostated, fresh saline solution and analysed by HPLC for drug content. At the end of the experiment $(24 \mathrm{~h})$, the skin surface was gently washed (3 times) with $1 \mathrm{ml}$ of distilled water, then dried with filter paper. The stratum corneum was removed by stripping with adhesive tape Tesa $^{\circledR}$ AG (Hamburg, Germany), and epidermis was separated from dermis with a surgical scalpel. Skin strata were cut, placed each in a flask with methanol and sonicated for $2 \mathrm{~min}$ in an ice bath to extract the drug. The tapes and tissue suspensions were filtered out and assayed for drug content by HPLC.

\subsection{In vitro biocompatibility of samples using keratinocytes}

Human keratinocytes, at passages 4-5, were grown as monolayer in $75 \mathrm{~cm}^{2}$ flasks, incubated in $100 \%$ humidity and $5 \% \mathrm{CO}_{2}$ at $37{ }^{\circ} \mathrm{C}$, using RPMI1640 supplemented with foetal bovine serum, penicillin/streptomycin and fungizone, as culture medium. For toxicity studies, cells were seeded into 96-well plates $\left(7.5 \times 10^{3}\right.$ cells/well $)$ and, after $24 \mathrm{~h}$, were treated for 24 and $48 \mathrm{~h}$ with quercetin coarse dispersion and quercetin nanosuspensions at different dilutions corresponding to 250, $100,50,25 \mu \mathrm{g} / \mathrm{ml}$ of drug. After incubation, cells were washed 3 times with fresh medium and their viability was determined by the MTT [3(4,5-dimethylthiazolyl-2)-2, 5-diphenyltetrazolium bromide] colorimetric assay, adding $200 \mu \mathrm{l}$ of MTT reagent $(0.5 \mathrm{mg} / \mathrm{ml}$ in PBS) to each well [21]. After $2-3 \mathrm{~h}$, the formed formazan crystals were dissolved in DMSO and their concentration was spectrophotometrically quantified at $570 \mathrm{~nm}$ with a microplate reader (Synergy 4, Reader BioTek Instruments, AHSI S.P.A, Bernareggio, Italy). All experiments were repeated at least three times. Results are shown as percent of cell viability in comparison with non-treated control cells (100\% viability).

\subsection{In vitro antioxidant activity of samples against induced $\mathrm{H}_{2} \mathrm{O}_{2}$ oxidative stress on keratinocytes}

Antioxidant activity was evaluated treating the cells with quercetin dispersion or quercetin nanosuspension $(100 \mu \mathrm{g} / \mathrm{ml}$ of quercetin) and simultaneously with hydrogen peroxide (1:30000 dilution) for $6 \mathrm{~h}$. After incubation, cells were washed 3 times with fresh medium and their viability was determined by the MTT [3(4,5-dimethylthiazolyl-2)2, 5-diphenyltetrazolium bromide] colorimetric assay, adding $200 \mu \mathrm{l}$ of MTT reagent $(0.5 \mathrm{mg} / \mathrm{ml}$ in PBS) to each well [21]. After $2-3 \mathrm{~h}$, the formed formazan crystals were dissolved in DMSO and their concentration was spectrophotometrically quantified at $570 \mathrm{~nm}$ with a microplate reader (Synergy 4, Reader BioTek Instruments, AHSI S.P.A, Bernareggio, Italy). All experiments were repeated at least three times. Results are shown as percent of cell viability in comparison with nontreated control cells (100\% viability).

\subsection{Statistical analysis of data}

Results are expressed as the mean \pm standard deviation and significance was tested at the 0.05 level of probability (p). For size, zeta potential, viscosity, drug accumulation and cytotoxicity, analysis of variance (ANOVA) was used to substantiate statistical differences between groups while Student's t-test was used for comparison between two samples using XLStatistic for Excel.

\section{Results and discussion}

\subsection{Nanocrystal characterization}

In this study, aiming at improving the bioavailability and the therapeutic activity of quercetin applied on the skin, nanosuspensions were formulated and used as an innovative approach. A pre-formulation study was performed to find out the best operating conditions for the preparation of nanosuspensions, by monitoring the variation of average size and polydispersity index (P.I.) of nanocrystals as a function of the milling time. Increasing the milling time, up to $40 \mathrm{~min}$, a reduction of both size and polidispersity index was observed. On the contrary, no further reduction of nanocrystal size was detected by increasing the milling time over $40 \mathrm{~min}$. Therefore, nanosuspensions were prepared maintaining the milling for $40 \mathrm{~min}$ at $3000 \mathrm{rpm}$. Four different formulations were obtained, and coarse suspension and quercetin water dispersion were also prepared and used as references (Table 1).

All nanoformulations were characterized for size, size distribution, surface charge, stability on storage, amorphous or crystalline form, solubility and all the tested physico-chemical properties were compared with those of a coarse suspension used as reference.

Generally, the main function of the stabilizer (whatever is the nature) is the improvement of the wettability of the hydrophobic surfaces of the drug particles, thus preventing their aggregation, by increasing the activation energy of the process $[10,22]$. In this work a non ionic surfactant and a polymer (i.e. T80 and P188 respectively), were used as stabilizer. When T80 was used as stabilizer, increasing the amount of quercetin from $3 \%$ to $5 \%$, both size and polydispersity index of nanocrystals decreased from $\sim 431 \mathrm{~nm}$ to $\sim 362 \mathrm{~nm}$ and from 0.33 to 0.26 respectively. On the contrary, P188 was able to better stabilize the system with the lowest amount of quercetin, being the mean diameter of nanocrystals approximately $\sim 326 \mathrm{~nm}$ versus $\sim 474 \mathrm{~nm}$ when the highest amount of drug was used (Table 2). However, overall results indicated that both T80 and P188 were effective on stabilizing small crystals, even when high amounts of drug were used.

To evaluate the stability of the systems, nanosuspensions were stored at room temperature $\left(\sim 25 \pm 1{ }^{\circ} \mathrm{C}\right)$ for 90 day. Size, size distribution and surface charge of nanocrystals were monitored over this period. As can be seen in Fig. 1, any significant increase on nanocrystals size was detected for T80 stabilized nanosuspensions, regardless of the amount of drug used.

A larger variation of particle size was evident during storage of P188 stabilized formulations. In particular, NS5 P188 (5\% quercetin) showed a significant increase of nanocrystal size $(\sim 27 \%)$ after only one day, then size remained constant up to 30 days, and finally from 60 to 90 days size and size distribution slightly decreased (Fig. 1).

Table 2

Mean diameter (MD), polydispersity index (PI) and zeta potential (ZP) of quercetin nanosuspensions (NS) and coarse suspensions (CS). Mean values \pm standard deviation (SD) obtained from at least 3 independent samples were reported.

\begin{tabular}{llll}
\hline & Size \pm SD & PI \pm SD & Zeta Potential \pm SD \\
\hline NS3 P188 & $326 \pm 24$ & $0.30 \pm 0.02$ & $-33 \pm 3$ \\
NS5 P188 & $474 \pm 30$ & $0.22 \pm 0.02$ & $-39 \pm 6$ \\
NS3 T80 & $431 \pm 15$ & $0.33 \pm 0.01$ & $-31 \pm 1$ \\
NS5 T80 & $362 \pm 26$ & $0.26 \pm 0.04$ & $-40 \pm 1$ \\
CS3 P188 & $4627 \pm 598$ & $0.70 \pm 0.3$ & $-45 \pm 2$ \\
CS5 P188 & $4040 \pm 875$ & $0.62 \pm 0.4$ & $-48 \pm 1$ \\
CS3 T80 & $2106 \pm 112$ & $0.73 \pm 0.5$ & $-45 \pm 1$ \\
CS5 T80 & $5836 \pm 108$ & $0.89 \pm 0.7$ & $-47 \pm 2$ \\
\hline
\end{tabular}




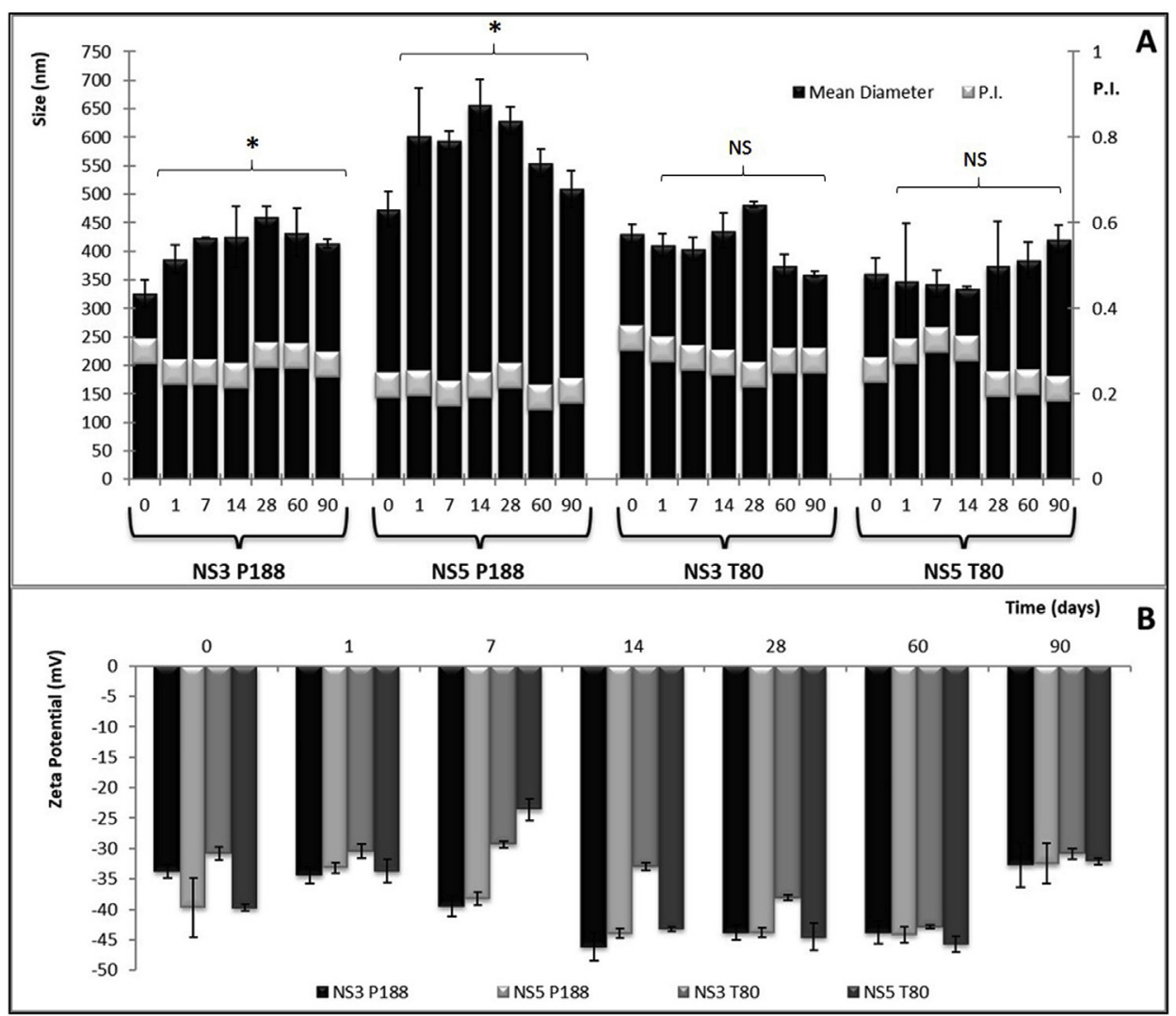

Fig. 1. Variation of mean diameter, polidispersity index and zeta potential of quercetin nanosuspensions during 90 days of storage at room temperature $\left(\sim 25 \pm 1{ }^{\circ} \mathrm{C}\right)$. Mean values \pm standard deviation (SD) are reported $(\mathrm{n}=6)$.

\subsection{Solid state characterization}

In order to evaluate the effects of formulation processes on drug crystalline properties, the solid state of bulk quercetin and freeze-dried quercetin nanocrystals were investigated using the X-Ray Powder Diffraction (XRPD) technique. Quercetin showed a semi-crystalline pattern with typical reflection peaks at $4.54^{\circ}, 9.78^{\circ}, 13.06^{\circ}, 26.00^{\circ}$, $27.92^{\circ} 2 \theta$ [23]. In the diffractograms of freeze-dried nanocrystals some diffraction peaks due to the crystalline structure of quercetin were still detected at a diffraction angle of $4.48^{\circ}, 12.42^{\circ}, 27.42^{\circ} 2 \theta$.

The FTIR spectra of bulk quercetin, freeze-dried nanocrystals and excipients were collected. Bulk quercetin showed the strong $\mathrm{O}-\mathrm{H}$ deformation band in the region between 3400 and $3000 \mathrm{~cm}^{-1}$ partially overlapped to the Csp2-H stretching, and at 1672, 1615 and $1513 \mathrm{~cm}^{-1}$ the $\mathrm{C}=\mathrm{O}$ and $\mathrm{C}=\mathrm{C}$ stretchings, respectively. The FTIR spectrum of all lyophilized quercetin nanocrystals are almost superimposable upon that of bulk quercetin; in addiction the absorption bands at 2920, 2852, 1729 attributable to $\mathrm{C}-\mathrm{H}$ and $\mathrm{C}=\mathrm{O}$ T80 and at $1112 \mathrm{~cm}^{-}{ }^{1} \mathrm{C}-\mathrm{O}$ P188 stretchings are also visible.

In Fig. 2, the FTIR spectra of bulk quercetin, P188 and NS3 P188 freeze-dried nanosuspension in the region between 1750 and $750 \mathrm{~cm}^{-1}$ are reported. In the formulation spectra the typical quercetin attributable to $\mathrm{C}=\mathrm{O}$ and $\mathrm{C}=\mathrm{C}$, were maintained. The peak at $1112 \mathrm{~cm}^{-1}$ belonging to $\mathrm{C}-\mathrm{O} \mathrm{P} 188$ is also visible.

The bulk quercetin DSC analysis presented a sharp endothermic peak at $318{ }^{\circ} \mathrm{C}$ (onset temperature) while the freeze-dried nanocrystals thermogram showed a melting peak at an onset temperature of $307.12{ }^{\circ} \mathrm{C}$, slightly shifted to lower temperature due to loss of crystallinity and/or for the presence of the formulation.

\subsection{Solubility studies}

Among flavonoids, quercetin $\left(3,3^{\prime}, 4^{\prime}, 5,7\right.$-pentahydroxylflavone) is the most representative compound of the flavonol subclass. However, its solubility in water is very low $(\sim 4 \pm 0.3 \mu \mathrm{g} / \mathrm{ml})$ as experimentally obtained during this work. The dissolution profiles of freeze-dried quercetin nanosuspensions, in comparison with both quercetin coarse suspension and dispersion in water, are shown in Fig. 3.

Quercetin solubility in water was markedly enhanced when the nanosystems were used, as more than $55-60 \mu \mathrm{g} / \mathrm{ml}$ of the drug was dissolved between 4 and $72 \mathrm{~h}$ (Table 3 ). Conversely, the solubility of quercetin was $\sim 9$ and $6 \mu \mathrm{g} / \mathrm{ml}$ when coarse suspensions were tested at the same experimental conditions and using P188 and T80 as stabilizer, respectively. According to Freundlich- Ostwald equation, such a great increase in the solubility of quercetin formulated as nanosuspension could be attributed not only to the solubilizing and wetting action of the surfactant but also to the particle size reduction [24].

These results confirmed that the use of nanosuspension may significantly increase the dissolution rate and bioavailability of low bioavailable drugs [10].

\subsection{In vitro penetration and permeation studies}

The main goal of this study was to improve the bioavailability and therapeutic effectiveness of quercetin after topical application, promoting its interaction with the skin and in turn with keratinocytes. The in vitro accumulation and permeation studies of the different formulations were performed using Franz diffusion cells and newborn pig skin, which represent an ideal model as it is very similar to human skin. Fig. 4 shows the results obtained after $24 \mathrm{~h}$ of experiment, no transdermal permeation of quercetin was detected regardless of the formulation applied in the skin (quercetin water dispersion, coarse 


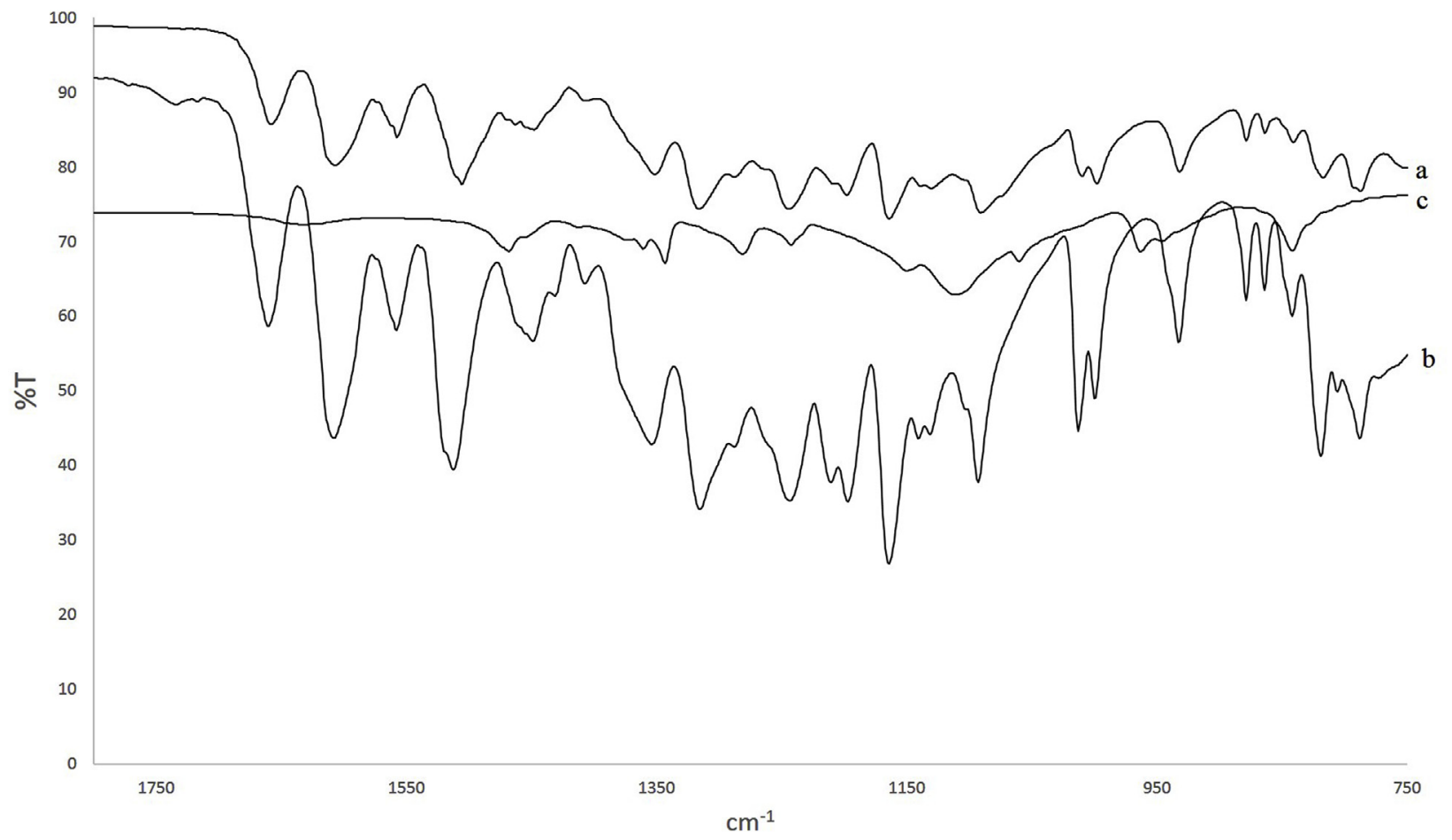

Fig. 2. Diffraction patterns of bulk quercetin (a), P188 (c) and NS3 P188 freeze-dried nanosuspension (b) in the region between 1750 and $750 \mathrm{~cm}^{-1}$.

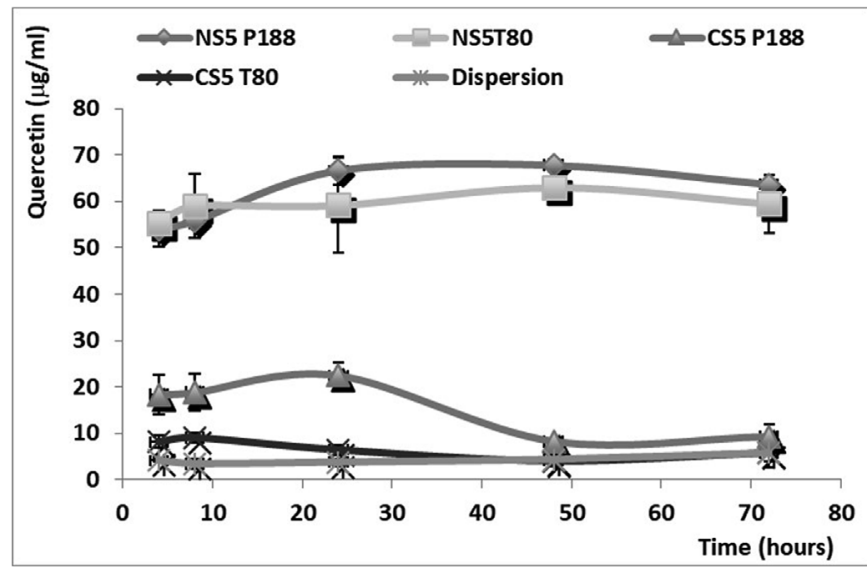

Fig. 3. Dissolution profiles of bulk quercetin dispersion, quercetin nanosuspension (NS5) and coarse suspension (CS5), both containing 5\% quercetin.

Table 3

Solubility in water of bulk quercetin, quercetin nanosuspension (NS) and coarse suspension (CS).

\begin{tabular}{ll}
\hline & Solubility $(\mu \mathrm{g} / \mathrm{ml} \pm \mathrm{SD})$ \\
\hline Bulk Quercetin & $4 \pm 1$ \\
NS5 P188 & $64 \pm 2$ \\
NS5 T80 & $60 \pm 6$ \\
CS5 P188 & $9 \pm 2$ \\
CS5 T80 & $6 \pm 3$ \\
\hline
\end{tabular}

suspensions or nanosuspension) [25,26]. Quercetin nanosuspensions containing T80 or P188 were able to favour the highest accumulation of the drug in the different skin strata within $24 \mathrm{~h}$ of application. The best results were obtained applying on the skin the nanosuspension containing the lowest amount of quercetin (3\%), when compared with the more concentrated nanosuspension (quercetin 5\%), as the percentage of drug detected in the different skin strata was $\sim 30$ and $\sim 18 \%$

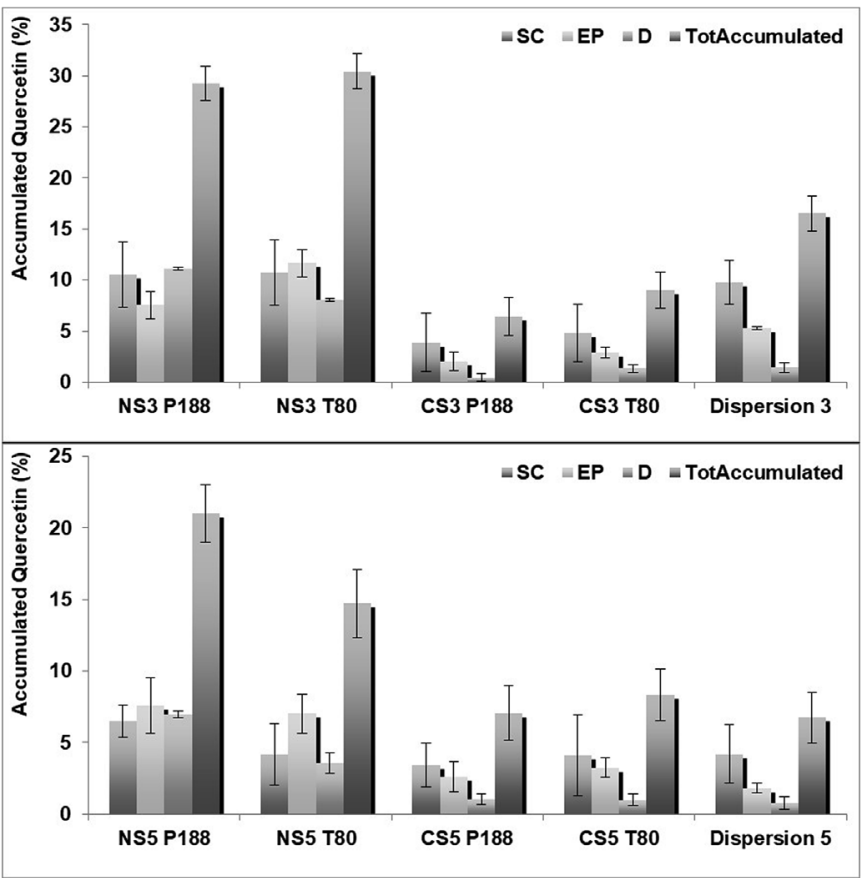

Fig. 4. Cumulative amount of drug accumulated in stratum corneum (SC), epidermis (EP) and dermis (D) after $24 \mathrm{~h}$ application of nanosuspension, coarse suspension and water dispersion of quercetin 3 and 5\% (upper and lower panel, respectively). Data represent the means \pm standard deviation (SD) of at least six experimental determinations.

respectively. In particular, nanosuspensions ensured a greater accumulation of quercetin especially in the stratum corneum and epidermis but also in the dermis. On the contrary, the application of quercetin in water dispersion or coarse suspension on the skin, did not promote the drug accumulation in the different skin strata, within $24 \mathrm{~h}$ experiment, as the $\%$ of drug detected was significantly lower and mainly confined 


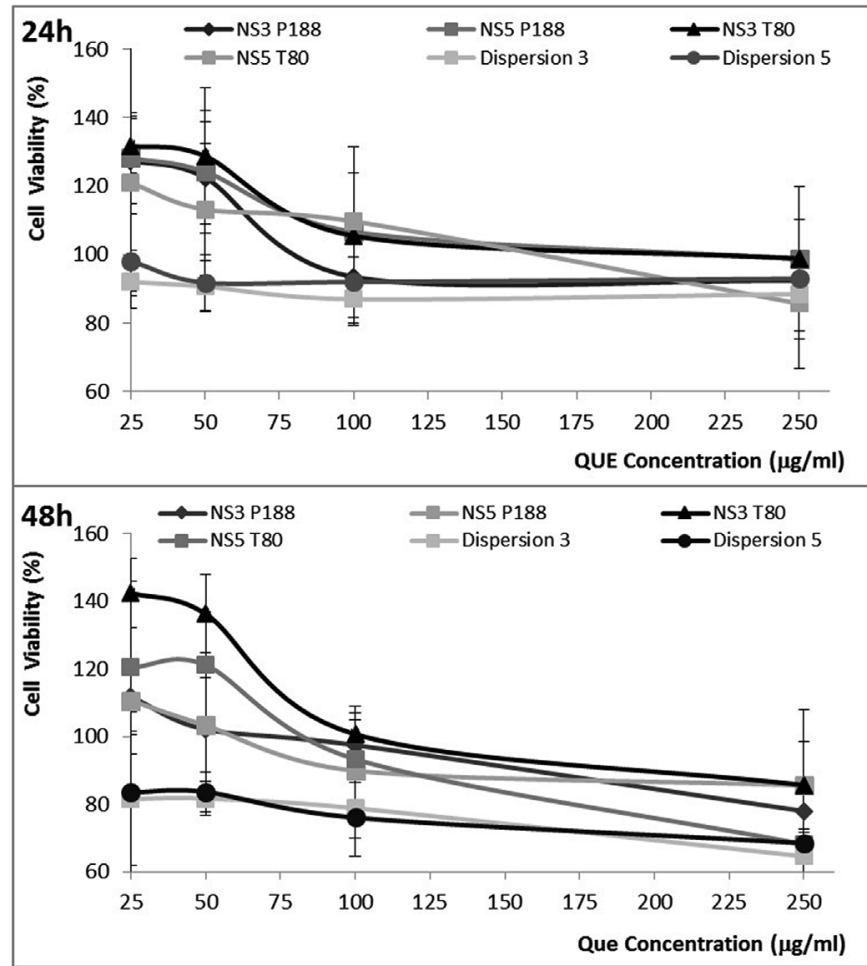

Fig. 5. In vitro cytotoxic effect of quercetin nanosuspension, coarse suspension and water dispersion on keratinocytes after 24 and $48 \mathrm{~h}$ of coincubation (upper and lower panel, respectively).

to the upper layer (i.e. stratum corneum), (Fig. 4).

The greater ability of nanosuspensions to promote the accumulation of quercetin in the skin strata, makes such formulations ideal systems for the treatment of topical diseases connected to inflammation or oxidative stress. The superior effectiveness of nanosuspension is probably related to the penetration enhancer effect of both T80 and P188, along with the reduced size of the crystals. In particular, the reduction of the crystals size led to their increased solubility and dissolution rate, producing a concentration gradient able to improve the penetration of the drug compared to the coarse suspensions or the quercetin dispersion in water.

\subsection{In vitro cytotoxicity studies on keratinocytes}

To evaluate the biocompatibility of quercetin nanosuspensions, an in vitro cytotoxicity study was performed using human keratinocytes and different concentration of the drug $(25,50,100$ and $250 \mu \mathrm{g} / \mathrm{ml}$ of quercetin) for 24 and $48 \mathrm{~h}$. As can be seen in Fig. 5, the nanosuspensions did not induce any toxic effect on keratinocytes exposed for 24 or $48 \mathrm{~h}$ to doses up to $100 \mu \mathrm{g} / \mathrm{ml}$ of quercetin. When the highest concentration of $250 \mu \mathrm{g} / \mathrm{ml}$ was employed, the nanosuspensions reduced the cell viability to around $82 \%$ after $48 \mathrm{~h}$, while a coarse suspension and a water dispersion exerted higher toxicity $(76 \%$ and $66 \%$ cell viability, respectively). Interestingly, the viability of cells treated with low doses of nanosuspensions ( $25 \mu \mathrm{g} / \mathrm{ml}$ quercetin) resulted to be promoted compared to the untreated controls. This phenomenon, more evident for T80-stabilized nanosuspensions, is probably connected to the well-known antioxidant activity of the active compound, which cell availability was improved as a consequence of the higher solubility of the nanocrystals. Overall data suggest that quercetin crystal size reduction to the nano scale resulted in highly biocompatible systems, T80-stabilized nanosuspensions having the lowest toxicity among the tested formulations.

\subsection{In vitro antioxidant activity of quercetin nanosuspension on keratinocytes}

Pharmaceutical effects of quercetin are mainly correlated to its high antioxidant activity. However, as reported before, its low water solubility is often associated to a poor bioavailability of the drug, which reduces its therapeutic effect. Encouraged by the results obtained by in vitro permeation studies, we evaluated the antioxidant effect of quercetin nanosuspensions in comparison with quercetin water dispersion, on keratinocytes stressed with a potent oxidant agent, the hydrogen peroxide, which cause high mortality of cells when added to the cell culture medium. As expected, the treatment of keratinocytes with hydrogen peroxide significantly reduced the cell viability (60\%) compared to untreated cells. The simultaneous treatment with quercetin water dispersion did not reduce the toxic/oxidative effect of hydrogen peroxide as the viability remained $\sim 60 \%$ regardless of the concentration used. Conversely, cells were protected from the toxic effect of the oxidative agent following the administration of quercetin nanosuspensions. Regardless of the formulation used (T80 or P188-stabilized nanosuspensions) a great protection of the cells was detected, as the viability was comparable to the control cells, not exposed to the hydrogen peroxide (Fig. 6).

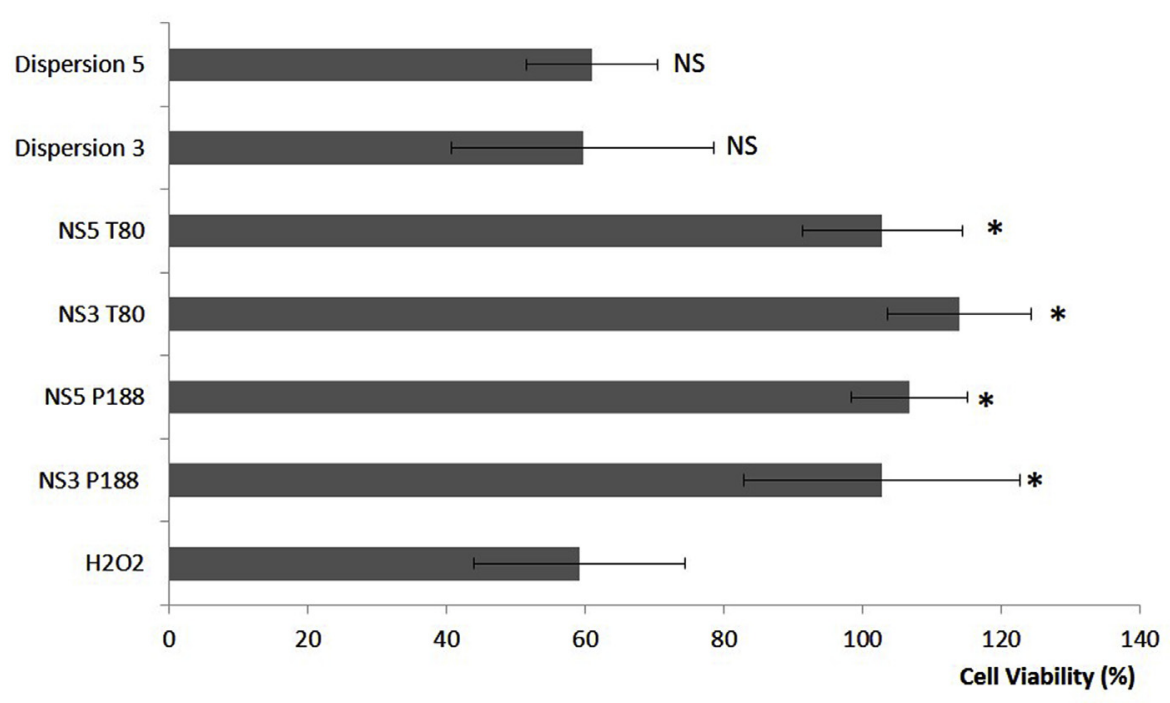

Fig. 6. In vitro antioxidant activity of quercetin nanosuspension and water dispersion on keratinocytes. 


\section{Conclusions}

Overall results obtained in this work confirmed that the formulation of quercetin as nanosuspension led to the formation of small crystals stabilized by using T80 or P188. Both nanosuspension were small in size and able to significantly increase the solubility and dissolution rate of quercetin, thus increasing the accumulation in the different skin layers, as well as the in vitro bioavailability in human keratinocytes. Moreover, the crystal size reduction to the nano scale resulted in highly biocompatible systems, able to protect cultured cells from an externally applied oxidative stress. Noteworthy, the developed nanosuspensions were able to improve skin accumulation without resulting in transdermal penetration. Therefore, in addition to the potential relevance for the treatment of cutaneous disorders, an application in the cosmetic field where the systemic exposure to the drug has to be prevented, could also be foreseen for these systems.

\section{Declaration of competing interest}

The authors declare that they have no known competing financial interests or personal relationships that could have appeared to influence the work reported in this paper.

\section{Appendix A. Supplementary data}

Supplementary data to this article can be found online at https:// doi.org/10.1016/j.jddst.2019.101482.

\section{References}

[1] A. Svobodová, J. Psotová, D. Walterová, Natural phenolics in the prevention of UVinduced skin damage. A review, Biomed. Pap. Med. Fac. Univ. Palacky. Olomouc. Czech. Repub. 147 (2003) 137-145.

[2] B.S. Nayak, D.D. Ramdath, J.R. Marshall, G.N. Isitor, M. Eversley, S. Xue, et al., Wound-healing activity of the skin of the common grape (Vitis Vinifera) variant, Cabernet Sauvignon, Phytother Res. 24 (2010) 1151-1157, https://doi.org/10. 1002/ptr.2999.

[3] S.R. Pinnell, Cutaneous photodamage, oxidative stress, and topical antioxidant protection, J. Am. Acad. Dermatol. 48 (2003) 1-22, https://doi.org/10.1067/mjd. 2003.16.

[4] R.J. Nijveldt, E. van Nood, D.E. van Hoorn, P.G. Boelens, K. van Norren, P.A. van Leeuwen, Flavonoids: a review of probable mechanisms of action and potential applications, Am. J. Clin. Nutr. 74 (2001) 418-425.

[5] F. Mainini, A. Contini, D. Nava, P.A. Corsetto, A.M. Rizzo, E. Agradi, et al, Synthesis, molecular characterization and preliminary antioxidant activity evaluation of quercetin fatty esters, J. Am. Oil Chem. Soc. 90 (2013) 1751-1759, https:// doi.org/10.1007/s11746-013-2314-0.

[6] F. Lai, I. Franceschini, F. Corrias, M.C. Sala, F. Cilurzo, C. Sinico, et al., Maltodextrin fast dissolving films for quercetin nanocrystal delivery. A feasibility study, Carbohydr. Polym. 121 (2015) 217-223, https://doi.org/10.1016/j.carbpol.2014 11.070.

[7] M.L. Manca, I. Castangia, C. Caddeo, D. Pando, E. Escribano, D. Valenti, et al., Improvement of quercetin protective effect against oxidative stress skin damages by incorporation in nanovesicles, Colloids Surfaces B Biointerfaces 123 (2014) 566-574, https://doi.org/10.1016/j.colsurfb.2014.09.059.

[8] L. Tavano, R. Muzzalupo, N. Picci, B. de Cindio, Co-encapsulation of lipophilic antioxidants into niosomal carriers: percutaneous permeation studies for cosmeceutical applications, Colloids Surfaces B Biointerfaces 114 (2014) 144-149, https://doi.org/10.1016/j.colsurfb.2013.09.055

[9] M. Chessa, C. Caddeo, D. Valenti, M. Manconi, C. Sinico, A.M. Fadda, Effect of penetration enhancer containing vesicles on the percutaneous delivery of quercetin through new born pig skin, Pharmaceutics 3 (2011) 497-509.

[10] P. Kocbek, S. Baumgartner, J. Kristl, Preparation and evaluation of nanosuspensions for enhancing the dissolution of poorly soluble drugs, Int. J. Pharm. 312 (2006) 179-186.

[11] R.H. Müller, S. Gohla, C.M. Keck, State of the art of nanocrystals - special features, production, nanotoxicology aspects and intracellular delivery, Eur. J. Pharm. Biopharm. 78 (2011) 1-9.

[12] F. Lai, M. Schlich, R. Pireddu, F. Corrias, A.M. Fadda, C. Sinico, Production of nanosuspensions as a tool to improve drug bioavailability: focus on topical delivery, Curr. Pharmaceut. Des. 21 (2015) 6089-6103.

[13] C.M. Keck, R.H. Müller, Drug nanocrystals of poorly soluble drugs produced by high pressure homogenisation, Eur. J. Pharm. Biopharm. 62 (2006) 3-16.

[14] I. Ghosh, B. Michniak-Kohn, Influence of critical parameters of nanosuspension formulation on the permeability of a poorly soluble drug through the skin-a case study, AAPS PharmSciTech 14 (2013) 1108-1117.

[15] E. Merisko-Liversidge, G.G. Liversidge, E.R. Cooper, Nanosizing: a formulation approach for poorly-water-soluble compounds, Eur. J. Pharm. Sci. 18 (2003) 113-120.

[16] A. Dolenc, J. Kristl, S. Baumgartner, O. Planinsek, Advantages of celecoxib nanosuspension formulation and transformation into tablets, Int. J. Pharm. 376 (2009) 204-212.

[17] G.B. Romero, R. Chen, C.M. Keck, R.H. Müiller, Industrial concentrates of dermal hesperidin smartCrystals $\left.{ }^{\circledR}\right)$ - production, characterization \& long-term stability, Int. J. Pharm. (2014).

[18] R. Pireddu, C. Sinico, G. Ennas, F. Marongiu, R. Muzzalupo, F. Lai, et al., Novel nanosized formulations of two diclofenac acid polymorphs to improve topical bioavailability, Eur. J. Pharm. Sci. 77 (2015) 208-215.

[19] R. Pireddu, C. Caddeo, D. Valenti, F. Marongiu, A. Scano, G. Ennas, et al., Diclofenac acid nanocrystals as an effective strategy to reduce in vivo skin inflammation by improving dermal drug bioavailability, Colloids Surfaces B Biointerfaces 143 (2016) 64-70, https://doi.org/10.1016/j.colsurfb.2016.03.026.

[20] F. Corrias, M. Schlich, C. Sinico, R. Pireddu, D. Valenti, A.M. Fadda, et al., Nile red nanosuspensions as investigative model to study the follicular targeting of drug nanocrystals, Int. J. Pharm. 524 (2017), https://doi.org/10.1016/j.ijpharm.2017. 03.042 .

[21] M. Letizia, M. Zaru, M. Manconi, F. Lai, D. Valenti, C. Sinico, et al., Glycerosomes : a new tool for effective dermal and transdermal drug delivery, Int. J. Pharm. 455 (n. d.) 66-74.

[22] B. Van Eerdenbrugh, G. Van den Mooter, P. Augustijns, Top-down production of drug nanocrystals: nanosuspension stabilization, miniaturization and transformation into solid products, Int. J. Pharm. 364 (2008) 64-75 http://www.sciencedirect. com/science/article/pii/S0378517308005280 accessed October 16, 2013.

[23] F. Lai, I. Franceschini, F. Corrias, M.C. Sala, F. Cilurzo, C. Sinico, et al., Maltodextrin fast dissolving films for quercetin nanocrystal delivery. A feasibility study, Carbohydr. Polym. 121 (2015), https://doi.org/10.1016/j.carbpol.2014.11.070.

[24] R.H. Müller, K. Peters, Nanosuspensions for the formulation of poorly soluble drugs, Int. J. Pharm. 160 (1998) 229-237.

[25] K. Mitri, R. Shegokar, S. Gohla, C. Anselmi, R.H. Müller, Lutein nanocrystals as antioxidant formulation for oral and dermal delivery, Int. J. Pharm. 420 (2011) 141-146, https://doi.org/10.1016/j.ijpharm.2011.08.026.

[26] R.H. Müller, M. Radtke, S.A. Wissing, Solid lipid nanoparticles (SLN) and nanostructured lipid carriers (NLC) in cosmetic and dermatological preparations, Adv. Drug Deliv. Rev. 54 (2002) S131-S155, https://doi.org/10.1016/S0169-409X(02) 00118-7. 